\title{
A Link Between Components of the Nasal Microbiome and an Individual's Susceptibility to Influenza
}

\author{
Alexx P. Weaver ${ }^{1}$, Sophia McClain ${ }^{1}$ and Whitney M. Holden ${ }^{1}$ \\ ${ }^{1}$ The Arkansas School for Mathematics, Sciences and the Arts, Hot Springs National Park, AR, USA \\ DOI: https://doi.org/10.47611/jsrhs.v10i2.1455
}

\section{$\underline{\text { ABSTRACT }}$}

According to CDC influenza estimates, the flu infects $\sim 40$ million people and causes 24,000 to 60,000 deaths in the United States annually. Vaccination can be highly effective but is often a neglected tool for preventing infection. In this project, three methods were developed to compare an individual's reported self-history of influenza infection to the types and amounts of nasal bacteria collected by nasal swab to assess if certain bacteria may correlate with less history of influenza infection. These three methods quantified species from four genera of bacteria - Staphylococcus, Micrococcus, Streptococcus and Haemophilus - and compared the amounts of each type of bacteria with participant survey answers regarding their history of influenza infection. In Method 1, a disk diffusion test with bacitracin distinguished isolates of Staphylococcus from Micrococcus. Higher ratios of Staphylococcus to Micrococcus were found in individuals less susceptible to influenza $(\mathrm{p}=0.003)$. In Method 2, S. pyogenes and S. pneumoniae were distinguished based on their hemolytic patterns. A higher proportion $S$. pneumoniae significantly correlated with more history of influenza $(\mathrm{p}=0.002)$. In Method 3, total numbers of Staphylococcus spp. and $H$. influenzae were compared. More frequent $H$. influenzae significantly correlated with higher influenza frequency $(\mathrm{p}=0.006)$. While all three methods indicate correlations between specific nasal bacteria and influenza susceptibility, Method 2 was the simplest and least expensive to perform. Commercialization of one or more of these methods could result in a simple and inexpensive test to identify at-risk individuals for influenza.

\section{Introduction}

The global burden of respiratory infections is very large, with more than 1.5 million deaths each year. Within the population of the United States alone, there are approximately 500 million respiratory illnesses each year, which costs the healthcare system $\$ 40$ billion USD annually. Influenza is one such viral respiratory infection that affects 1 billion people worldwide every year and causes 300,000-500,000 deaths annually (Reich et al., 2019). Influenza infections affect most populations but are especially deadly for the very young, elderly, and those with lung disease (RyndaApple et al., 2015). Influenza begins in the upper respiratory tract and then further spreads throughout the body (Chen et al., 2016). The virus has many strains and sub-strains that mutate and appear each year with the most popular strains being influenza A and influenza B. Influenza A is zoonotic with the ability to infect both animals and humans. These characteristics allow it, when passed from animal to human hosts, to mutate quickly and be highly infectious, leading to large influenza epidemics like avian or swine flu. Meanwhile, influenza B is not able to infect animals and only survives in human hosts. Due to this characteristic, it is not responsible for larger epidemics (Liu et al., 2015).

Healthy humans have a diverse microflora that generally has a symbiotic and asymptomatic relationship with their hosts. Perhaps the most well-known of these symbiotic microflora is the gastrointestinal microbiome, a diverse, thriving community of bacteria that can be strengthened and balanced by the use of probiotics. The popularity of probiotics is rising each year with one in five Americans taking probiotics and a booming global market worth $\$ 15$ billion USD annually (Day et al., 2019; Wilkins \& Sequoia, 2017). The most common types of over-the-counter probiotics are dietary supplements contain Lactobacillus, Bifidobacterium, and Saccharomyces species (Wilkins \& 
Sequoia, 2017). Within the medical community, there has also been a rise in the popularity of fecal microbiota transplantations as treatment for chronic digestive disorders such as inflammatory bowel disease, irritable bowel syndrome, and even as treatment for some foodborne allergies. Fecal microbiota transplantations directly introduce a healthy microbiome to the patient and allows for a rapid and near complete restoration of their digestive microbiome (Zeng et al., 2019). In addition to the gastrointestinal microbiome, there are known mutualistic symbionts in many compartments of the human body. The most common nasal bacteria of this type include commensal Staphylococcus, Haemophilus influenzae, Micrococcus spp., Streptococcus pneumoniae, and Streptococcus pyogenes. The carriage for these bacteria is much higher in children and youth when compared to adults because of the close contact environments — such as daycares, schools, and college dorms — that these age groups experience (Pan et al., 2016). There is evidence that probiotics can be used as preventative therapy for upper respiratory infections due to their ability to aggregate and compete with pathogens for nutrients and space (Popova et al., 2012).

Because of the compromised immune system that occurs during an influenza infection, the risk of secondary bacterial infections increases, leading to superinfections. Superinfections decrease the efficiency of the macrophages and neutrophils (white blood cells that help fight infection via a process called phagocytosis) which greatly increases the severity of the disease. Bacterial superinfection contributed to higher morbidity and mortality during the 2009 influenza pandemic (Rynda-Apple et al., 2015). The combination of influenza and Staphylococcus (most commonly S. epidermidis or S. aureus) is one example of bacterial superinfection. According to the National Institutes of Health, staphylococci account for most of the biofilm-based human infections (Paharik \& Horswill, 2016). The Staphylococcus genus is a large part of the human microflora, and it usually exists in a peaceful relationship with its host. In most cases, Staphylococcus is not virulent and does not cause harmful infections (Otto, 2009). One of the most common staphylococci species, found in $96 \%$ of healthy people, is S. epidermidis (Chen et al., 2016). S. epidermidis is a grampositive bacterium found typically on the skin and nose and is a part of the coagulase-negative staphylococci. It is a non-motile and non-spore-forming bacterium that rarely causes infection (Namvar et al., 2014). Another common member of this genus, Staphylococcus aureus is a gram-positive round-shaped bacterium found in the human respiratory tract, nasal cavity, and on the surface of the skin (Otto, 2009). 20\% - 30\% of the world's population carry $S$. aureus in their noses, where the bacteria colonize in the anterior part of the nose and can cause infections in some hosts while remaining non-pathogenic in others (Warnke et al., 2014). Colonies of S. epidermidis have also been linked to significantly reduced amounts of $S$. aureus colonization which helps to reduce harmful infections in the body by $S$. aureus (Chen et al., 2016).

One possible mechanism of microbiome-mediated protection of a host from influenza involves the Staphylococcus Embp biofilm protein. Staphylococcus species easily attach to surfaces and form strong multicellular communities known as biofilms. The formation of a biofilm occurs in three distinct stages: attachment, accumulation (maturation), and detachment (dispersion) (Büttner et al., 2015; Otto, 2009; Rabin et al., 2015). To properly form a biofilm, Staphylococcus requires specific proteins and molecules. One example is Embp (extracellular matrix binding protein), a fibrinogen-binding protein found in $90 \%$ of all $S$. epidermidis strains that is integral for biofilm formation and function (Linnes et al., 2013). Due to the strong attachments formed in biofilm structures, bacteria can become resistant to the host's immune clearing system, meaning that these structures are long lasting formations (Rabin et al., 2015). Staphylococcus, with the function of Embp, significantly suppresses the infectivity of the influenza virus in chicken embryos and enhanced survival when the embryos were infected with influenza (Chen et al., 2016). Embp from Staphylococcus biofilm matrices can filter influenza viruses in human noses and even bind to influenza directly, indicating the likelihood for a key role in fighting influenza infections (Chen et al., 2016; Linnes et al., 2013).

Staphylococcus species are not the only nasal commensal bacteria to consider when searching for a link between certain microbiota and a lowered risk of influenza infection. Within the nasal cavity of healthy individuals, there are also large quantities of Micrococcus (Becker et al., 2015; Cole et al., 1999). Micrococcus is a gram- and catalase-positive cocci bacteria. They are small non-motile, spherical cells that arrange themselves into clusters or bunches (Becker et al., 2015). In the case of distinguishing between Staphylococcus and Micrococcus in a culturebased assay, a disk diffusion test with bacitracin can accurately differentiate between the two genera when grown on 
mannitol salt agar (MSA) (Johnson et al., 1945). Staphylococcus has gained high resistance to bacitracin, while $M i$ crococcus remains highly susceptible. This difference in bacitracin resistance allows a bacitracin disk assay to serve as an accurate test for distinguishing the genera of these bacteria when collected via nasal swab and grown in culture (Baker et al., 1986; Falk \& Guering, 1983).

Streptococcus is also a common genus found in the human respiratory tract. Asymptomatic colonization occurs in approximately $5 \%$ to $20 \%$ of healthy individuals (Patterson, 1996). Streptococci are Gram-positive, nonmotile, non-spore-forming, catalase-negative cocci that can be divided into three types based on hemolysis patterns on blood agar. In this study, we observed two distinct hemolytic types of streptococci, $\alpha$-hemolytic (incomplete, green hemolysis) and $\beta$-hemolytic (clear, complete lysis of red cells). S. pneumoniae is the most common $\alpha$-hemolytic streptococcus found in the respiratory tract flora (Patterson, 1996). This type of bacteria commonly colonizes the nasopharynx in biofilms (Marks et al., 2014). On the other hand, S. pyogenes is the most common $\beta$-hemolytic streptococcus in the nasopharynx of humans, but is even more commonly found on human skin. Both of these Streptococcus species are capable of causing severe infections, but can also persist asymptomatically as part of the typical human nasal microbiome (Marks et al., 2014). Selective Strep agar can be used to select for and differentiate S. pyogenes and S. pneumoniae species from nasal swabs.

Haemophilus influenzae is another bacterium commonly found in the human nasal cavity and respiratory tract (Haemophilus influenzae, CDC.gov, 2018). H. influenzae is a non-motile, anaerobic, Gram-negative coccobacillus, and is only prevalent in humans (Agrawal \& Murphy, 2011). Many types of H. influenzae appear to be predominantly asymptomatic and non-threatening while they reside in the human throat and nasal cavity. This bacterium, although generally commensal, can also cause respiratory illnesses like pneumonia (Haemophilus influenzae, CDC.gov, 2018). H. influenzae can sometimes form dangerous superinfections with S. aureus and S. pneumoniae. Such bacterial combinations have the ability to cause bacterial meningitis, which can be lethal (Rynda-Apple et al., 2015). Chocolate agar with bacitracin can be used to selectively culture $H$. influenzae while inhibiting the growth of other bacteria.

A study of these common nasal bacteria from nasal swabs of individuals with and without a previous history of influenza infection could create more effective methods for predicting an individual's susceptibility to influenza, allowing better identification of individuals at higher risk of contracting influenza, especially from otherwise healthy populations. The connections this project observed between influenza and nasal bacteria like Staphylococcus species, Micrococcus species, $H$. influenzae, S. pyogenes, and $S$. pneumoniae could potentially save lives across the globe by identifying people at higher risk for contracting influenza based on their unique nasal microbiome composition. To evaluate these relationships, this study developed and validated three methods of quantifying specific types of nasal bacteria and their correlation to previous influenza infection history in a study population of healthy high school students. Method 1 uses zones of inhibition found via a disk diffusion test with bacitracin on mannitol salt agar to distinguish Staphylococcus species from Micrococcus species to determine if a correlation exists between susceptibility and the frequency of these two genera collected from nasal swabs. Method 2 uses a type of blood agar called Selective Strep agar to determine if a correlation exists between influenza susceptibility and the frequency of $S$. $p y$ ogenes and S. pneumoniae, identified by their hemolytic patterns, collected from nasal swabs. Method 3 uses Selective Strep agar and Chocolate agar to determine if a correlation exists between susceptibility and the frequency of total Streptococcus species to $H$. influenzae. Each method was also assessed for cost, as well as effectiveness. Influenza vaccination history was also collected from participants to compare vaccination rates in this group with influenza outcomes.

\section{Methods}

\section{Study Setting:}

This study was conducted in a BSL-2 laboratory at the Arkansas School for Mathematics, Sciences and the Arts (ASMSA) from March 2019 through January 2020. It was a survey and laboratory-based study that involved 
commercial samples of Staphylococcus epidermidis and Micrococcus luteus, as well as multiple sample isolates of these genera along with Haemophilus influenzae, Streptococcus pyogenes, and Streptococcus pneumoniae obtained via selective and differential agar from participant nasal cavities.

\section{Inclusion/Exclusion Criteria:}

The inclusion factors in this study were sex (to create an equal and representative sample with males and females) and previous history of influenza infection (to have a sample population with roughly equal frequencies of past influenza infection and no history of past influenza infection). Age was an exclusion factor, with only students between the ages of 15 and 18 from ASMSA allowed to participate.

\section{Participants:}

The participants in this study were all students from the ASMSA school population whose ages ranged from 15 and 18 years old, were of diverse races/ethnicities, and of both sexes. An email was sent to the entire ASMSA student population to recruit participants. The email included a summary of the study and what would be required if they wished to participate. Students then responded to the email if they wished to participate. Those who responded were contacted and required to read and sign an informed consent form that explained their role and rights in the project. Parental consent was also required for any student under the age of 18 . The students were allowed to withdraw from the study at any time. The faculty mentor of this study collected the consent forms and stored them in a secure location. A unique identification number generated by the faculty mentor was given to each individual once the informed consent form was signed. Participants then completed a Google survey that included questions about race/ethnicity, sex, age, the frequency of influenza infection, and how many times in the past each participant had received the influenza vaccine. The student researchers did not know which ID number was assigned to each participant, so surveys were anonymized. The participants were placed into two groups (identified only by their anonymous ID numbers) based on survey responses: previous influenza infection history and no previous influenza infection history. Once they had received their anonymous identification number, students in both groups were instructed to visit a licensed medical professional (the school nurse) to have their noses swabbed. The participants came to the nurse's office at a time of their choosing for 15 minutes on one day to complete the nasal swab procedure.

\section{Nasal Swabbing Process:}

Each participant reported anonymously to the nurse's office for a nasopharynx swabbing. The nurse used a sterile, dry swab purchased from Flinn Scientific (https://www.flinnsci.com) to circulate each nasal cavity with light pressure. These swabs were placed in the original sterile packaging, sealed, and labeled with the individual's corresponding unique identification number, which was unknown to the student researchers to give participants anonymity. These sealed anonymous swab packages were placed into a collection box and collected at the end of the day by the student researchers.

\section{Agar Preparation and Storage:}

The agar types used within this study consisted of Mannitol Salt Agar (MSA), Selective Strep agar, and Chocolate agar with bacitracin. The MSA was ordered online from Carolina Biological Supply (https://www.carolina.com) and the Selective Strep agar and Chocolate agar with bacitracin were ordered online from Hardy Diagnostics (https://hardydiagnostics.com). Multiple $125 \mathrm{~mL}$ bottles of ready-to-use MSA were purchased, heated via boiling water, and poured into small (diameter $=47 \mathrm{~mm}$ ) petri dishes, purchased from Fisher Scientific (https://www.fishersci.com), with approximately $12 \mathrm{~mL}$ of Mannitol Salt Agar per poured plate. Each plate was covered to prevent contamination and left at room temperature to solidify after pouring. There were approximately 230 MSA plates used in this study to test isolates individually to distinguish them as a species of Staphylococcus or Micrococcus. There were 40 Chocolate agar with bacitracin and 40 Selective Strep agar plates used in this study, one of each type per 38 study participants, plus two of each type for negative controls, since all recovered isolates from one 
study participant could be identified on one plate. The Selective Strep agar plates and Chocolate agar with bacitracin plates were ordered as pre-poured, ready to use media plates (diameter $=100 \mathrm{~mm}$ ) and required no preparation before use. All plates were stored in the refrigerator at $4{ }^{\circ} \mathrm{C}$ to prevent their dehydration prior to use.

\section{Precursor Procedures:}

Student researchers wore sterile nitrile gloves during all procedures with bacteria. The lab bench work surface was sanitized with $70 \%$ ethanol prior to work. Sterile (aseptic) technique, taught to the student researchers by their advisor, was used throughout the procedures. One of each plate (MSA, Selective Strep agar, and Chocolate agar with bacitracin) was removed from the refrigerator and labeled with the date and the identification number corresponding to the nasal swab to be tested. In addition to these plates, two autoclaved and sterilized microcentrifuge tubes were labeled with the identification number. One microcentrifuge tube was filled with 1,000 $\mu \mathrm{l}$ of sterile water, and the other microcentrifuge tube was filled with $1,242 \mu \mathrm{l}$ of sterile water using calibrated micropipettes and sterile pipette tips. The nasal swab was placed into the microcentrifuge tube containing $1,000 \mu \mathrm{l}$ of sterile water and the swab stem was cut, via sterilized scissors, to fit into the container with a closed lid. The tube was gently vortexed on low speed for 30 seconds to resuspend the nasal bacteria within the sterile water. Then, $138 \mu \mathrm{l}$ of the resuspended solution containing nasal bacteria was pipetted into the second microcentrifuge tube containing $1,242 \mu \mathrm{l}$ of sterile water, resulting in 1,380 total $\mu \mathrm{l}$ at a 1:10 dilution from the original, to facilitate easier counting of eventual CFU on plates, which could have grown in lawns if plated without first diluting. This 1:10 dilution tube was gently vortexed on low speed for 30 seconds to thoroughly mix the solution of bacteria. From this second tube containing the 1:10 dilution nasal bacteria suspension, $250 \mu \mathrm{l}$ of the solution was plated onto a labeled MSA plate, $550 \mu \mathrm{l}$ was plated onto a labeled Selective Strep agar plate, and $550 \mu \mathrm{l}$ was plated onto a labeled Chocolate agar with bacitracin plate. The amount of nasal bacteria suspension plated on the MSA and other two agar varieties was different due to the size difference between these two plate types (diameters of $47 \mathrm{~mm}$ for MSA and $100 \mathrm{~mm}$ for the other two media types). Using different amounts of solution per differently-sized plates allowed approximately the same amount of the nasal bacteria suspension to be pipetted per square millimeter of agar. Once the nasal bacteria suspension was pipetted onto a plate, it was swirled in a circular motion and a sterile loop purchased from Carolina Biological Supply (https://www.carolina.com) was used to evenly distribute the prepared bacterial solution to completely cover the agar surface. The plates were incubated at $37^{\circ} \mathrm{C}$ for 24-48 hours. Following each nasal swab's use, it was placed into a beaker of bleach for 20 minutes and then transferred into a trash can.

\section{Method 1 - Assessing Micrococcus and Staphylococcus:}

After the incubation described above in the precursor procedures, the bacterial growth on each MSA plate was recorded by counting the number of colonies grown and recording the result as colony forming units (CFU). From this initial MSA plate, 3-5 random colonies were chosen per study participant and, using a sterile loop, inoculated onto their own individually labeled MSA plates. In the center of each inoculated plate, a 10-unit bacitracin antibiotic disk was placed using sterile forceps. These plates were incubated at $37^{\circ} \mathrm{C}$ for another $24-48$ hours. After the incubation period, the zone of inhibition on each plate was measured in millimeters with a ruler and recorded. Zones of inhibition allowed for distinction of each isolate as Staphylococcus or Micrococcus. Micrococcus is denoted by a zone of inhibition $>25 \mathrm{~mm}$ and Staphylococcus is denoted by a zone of inhibition of $\leq 25 \mathrm{~mm}$. After photographing each plate, all plates were autoclaved at $121{ }^{\circ} \mathrm{C}$ for 30 minutes before disposal. Autoclaving was conducted by the adult research mentor.

\section{Method 2 - Assessing S. pneumoniae and S. pyogenes:}

After the incubation described above in the precursor procedures, the bacterial growth on each Selective Strep agar plate was recorded by counting the total number of CFU present. Colonies from the Selective Strep agar were identified as $\alpha$-hemolytic $S$. pneumoniae if there was no hemolytic zone (no halo), or $\beta$-hemolytic S. pyogenes if there was a large hemolytic zone (halo). Unlike Method 1, no isolates were taken from the Selective Strep agar to be cultured 
separately for further study because this agar is selective and possesses the ability to distinguish between different bacteria types grown on the same plate. After photographing each plate, all plates were autoclaved at $121{ }^{\circ} \mathrm{C}$ for 30 minutes before disposal. Autoclaving was conducted by the adult research mentor.

\section{Method 3 - Assessing Streptococcus spp. and H. influenzae:}

After the incubation described above in the precursor procedures, the bacterial growth of each Chocolate agar with bacitracin plate was recorded by counting the number CFU present to give the number of $H$. influenzae cultured from each swab. Unlike Method 1, no isolates were taken from the Chocolate agar with bacitracin to be cultured separately for further study because this agar is selective and possesses the ability to select for only the desired bacteria. After photographing each plate, all plates were autoclaved at $121^{\circ} \mathrm{C}$ for 30 minutes before disposal. Autoclaving was conducted by the adult research mentor. Method 3 combined these CFU numbers for $H$. influenzae and the total number of CFU recorded per participant in Method 2, without distinguishing between species of Streptococcus based on hemolytic zones.

\section{Controls:}

The positive controls for the MSA agar were live bacterial samples of Staphylococcus epidermidis and Micrococcus luteus purchased from Carolina Biological Supply (https:/www.carolina.com). These live cultures were each swabbed onto separate MSA plates via a sterile loop and sealed, labeled, and incubated at $37^{\circ} \mathrm{C}$ for $24-48$ hours. They were then used to confirm the expected zone of inhibition sizes previously reported in the literature to confirm that the bacitracin assay on MSA was a sound way to distinguish between these two genera.

The Selective Strep agar and Chocolate agar with bacitracin plates were created to be both selective and differential in growing the target bacteria. The company guaranteed the agars' ability to select and differentiate based on their behavior in multiple control experiments. In addition to that, the colonies that grew on these plates in this study matched the phenotypic descriptions expected for the bacteria these plates were designed to select.

The negative controls in all culture experiments were separate Petri dishes filled with the three respective agars with no bacteria plated. Each plate was inoculated with sterile water without bacteria, sealed, labeled, and incubated at $37^{\circ} \mathrm{C}$ for $24-48$ hours alongside the experimental plates prepared at the same time. The purpose of these negative control plates was to ensure no CFU grew and that the water and tools used (loops, pipette tips, etc.) were indeed sterile.

\section{Statistical Analysis:}

In Method 1, a 2 x 2 Chi-Square Analysis (https://www.socscistatistics.com/tests/chisquare2/default2.aspx) compared the amounts of Staphylococcus and Micrococcus isolates observed in the two groups (previous history of influenza infection and no previous history of influenza infection). In this method, the null hypothesis was that the CFU would not differ significantly between the two groups. In this analysis, a p-value of $\mathrm{p}<0.05$ indicated a statistically significant difference between the groups.

In Method 2, a 2 x 2 Chi-Square Analysis compared the CFU of both S. pyogenes and S. pneumoniae in the previous influenza illness and no previous influenza categories, with the null hypothesis being that the CFU would not differ significantly between the two groups. In this analysis, a p-value of $\mathrm{p}<0.05$ indicated a statistically significant difference between the CFU of the two bacteria species.

In Method 3, a Chi-Square Analysis compared the total CFU of Staphylococcus spp. and H. influenzae in the previous influenza illness and no previous influenza categories, with the null hypothesis being that the amounts would not differ significantly between the two groups. In this analysis, a p-value of $\mathrm{p}<0.05$ indicated a statistically significant difference between the CFU of the two bacteria species.

To examine the most complete picture of the nasal microbiome composition possible in this study, a separate 4 x 2 Chi-Square Analysis was conducted comparing the CFU of all Staphylococcus/Micrococcus CFU (including the hundreds that could not be tested with bacitracin to distinguish their specific genus due to cost limitations), $S$. 
pyogenes, $S$. pneumoniae, and $H$. influenzae from each of the previous influenza history groups in a single analysis. The null hypothesis was that the CFU observed would not differ significantly between the two groups. In this analysis, a $\mathrm{p}$-value of $\mathrm{p}<0.05$ indicated a statistically significant difference between the groups.

\section{Results}

\section{Comparison of Different Types of Nasal Bacteria Using Selective Media}

Three types of selective and differential agar-based media were used in this study: mannitol salt agar (MSA), Chocolate agar with bacitracin, and Selective Strep agar (Figure 1). Each of these media are uniquely selective and differential when compared to one another; MSA selects for Staphylococcus and Micrococcus (Figure 1a, 1b), which can be further distinguished from each other by the introduction of a bacitracin disk (Figure 2), Chocolate agar with bacitracin selects for $H$. influenzae (Figure 1c, 1d), and Selective Strep agar selects for S. pyogenes and S. pneumonia (Figure 1e, 1f), which can be further distinguished from each other by hemolytic patterns (Figure 3).

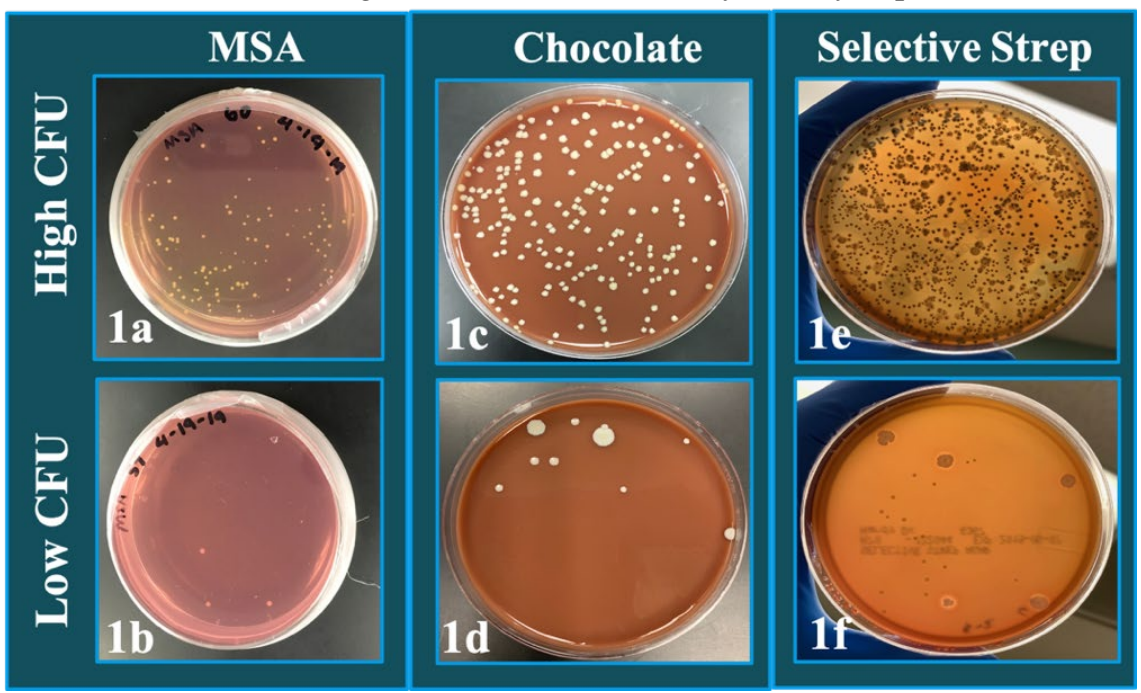

Figure 1. Representative images of bacterial growth on respective media types, measured in CFU, with example images of CFU numbers either in the top $10 \%(\mathbf{a}, \mathbf{c}, \mathbf{e})$ or bottom $10 \%(\mathbf{b}, \mathbf{d}, \mathbf{f})$ of samples to demonstrate the variable amounts of growth between nasal samples from different individuals. Three different types of selective and differential agar were used in this study: mannitol salt agar (MSA) (a, b), chocolate agar with bacitracin (c, d), and selective Strep $\operatorname{agar}(\mathbf{e}, \mathbf{f})$.

On MSA agar, 10-unit bacitracin disks were used to distinguish between Staphylococcus and Micrococcus based on their differing susceptibilities to this antibiotic (Figure 2). Micrococcus colonies demonstrate a zone of inhibition $>$ $25 \mathrm{~mm}$ while Staphylococcus colonies demonstrate a zone of inhibition $\leq 25 \mathrm{~mm}$. These published values (Baker et al., 1986; Falk \& Guering, 1983) were directly confirmed in this study by using commercially-available isolates of Micrococcus luteus and Staphylococcus epidermidis. Therefore, all isolates in this study with a zone of inhibition $>$ $25 \mathrm{~mm}$ were classified as Micrococcus and all isolates $\leq 25 \mathrm{~mm}$ were classified as Staphylococcus. 

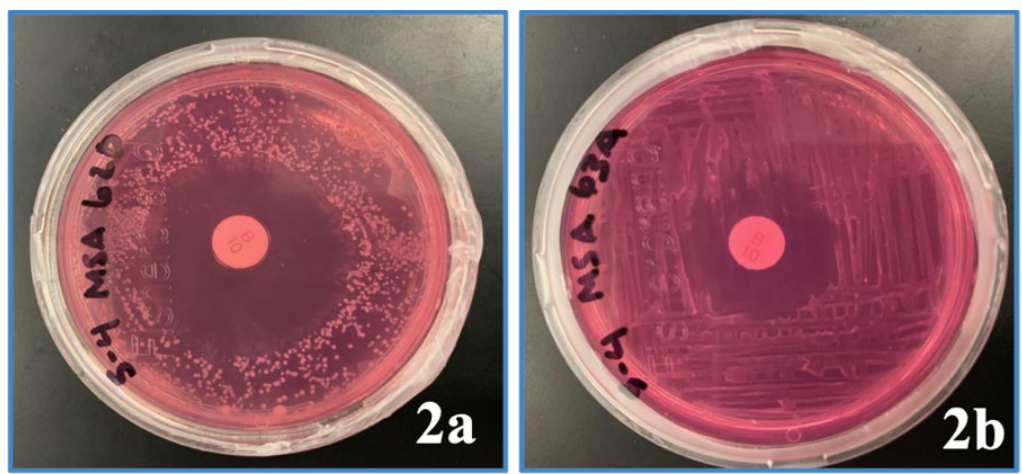

Figure 2. Example images of Staphylococcus and Micrococcus differences in bacitracin susceptibility. (a) Zones of inhibition $>25 \mathrm{~mm}$ were classified as Micrococcus. (b) Zones of inhibition $\leq 25 \mathrm{~mm}$ were classified as Staphylococcus.

Selective Strep agar isolates for two unique species of Streptococcus: S. pneumoniae and S. pyogenes. This differentiation between these bacteria is determined through investigation of alpha $(\alpha)$ and beta $(\beta)$ hemolytic patterns when grown on Selective Strep agar (Figure 3).
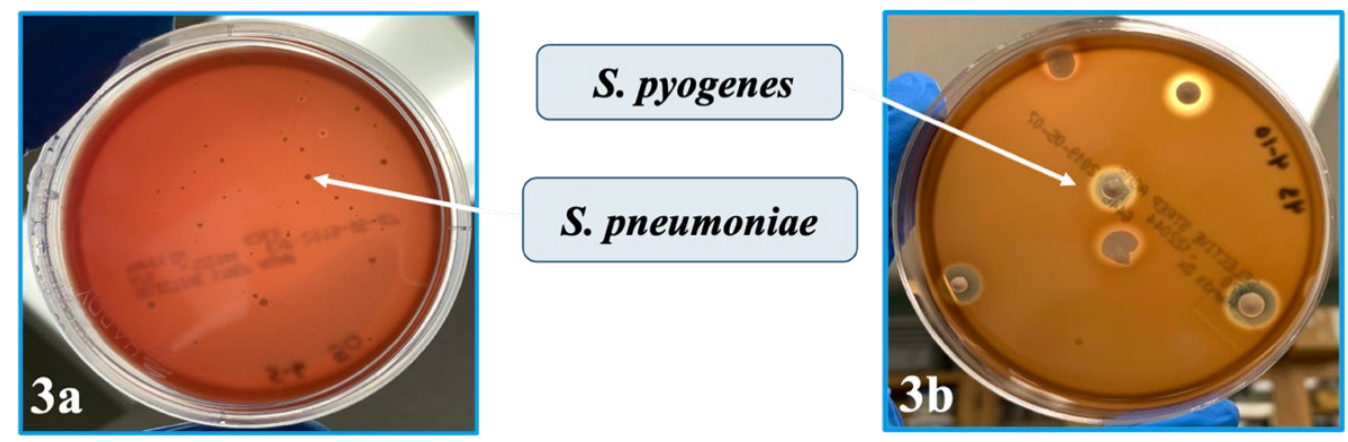

Figure 3. Representative images of hemolysis on Selective Strep agar. (A) S. pneumoniae colonies present without a halo, indicating a-hemolysis. (B) S. pyogenes colonies present with large halos, indicating $\beta$-hemolysis.

Using these three types of agar (Figure 1), the total CFU for Staphylococcus and Micrococcus species, S. pyogenes, $S$. pneumoniae, and $H$. influenzae collected from the test population were counted (Table 1). The frequencies and ratios of these bacteria were compared in the differing influenza history categories of Previous Influenza and No Previous Influenza by Chi-Square Analysis $(\mathrm{p}<0.00001)$, which demonstrated that the overall nasal flora correlates with influenza susceptibility. In terms of raw CFU counts, individuals who had self-reported zero previous influenza diagnoses had less CFU in the Staphylococcus and Micrococcus category, the S. pneumoniae category, and the $H$. influenzae category, and more CFU in the $S$. pyogenes category. The overall ratios also appear different, with bacteria in the Staphylococcus and Micrococcus category being overwhelmingly more dominant (70\%) in those with a history of influenza infection than those participants with no previous influenza diagnosis, who had more Streptococcus combined (52\%) than Micrococcus and Staphylococcus (37\%) (Figure 4).

Table 1. Nasal microbiota identified in groups with differing influenza infection histories

\begin{tabular}{|c|c|c|}
\hline Bacteria Type & Previous Influenza (n=21) & No Previous Influenza (n=17) \\
\hline Staphylococcus/Micrococcus & 536 & 82 \\
\hline S. pyogenes & 48 & 54 \\
\hline S. pneumoniae & 115 & 60 \\
\hline
\end{tabular}




\begin{tabular}{|c|c|c|}
\hline H. influenzae & 71 & 24 \\
\hline \multicolumn{2}{|c|}{ Chi-Square statistic: 99.60} & $\mathrm{p}<0.00001$ \\
\hline
\end{tabular}

Table 1. The average CFU of these bacteria, rounded to the nearest whole number, per participant in the "previous influenza" group and the "no previous influenza" group, as self-reported by participants. This indicates that levels of these types of bacteria in a nasal microbiome correlate with frequency of influenza illness. These CFU were plated from a 1:10 dilution, so the original number of bacteria recovered from the nasal swabs is estimated to be 10x higher. Zero CFU grew on negative control plates (not shown), indicating success in following sterile procedure.
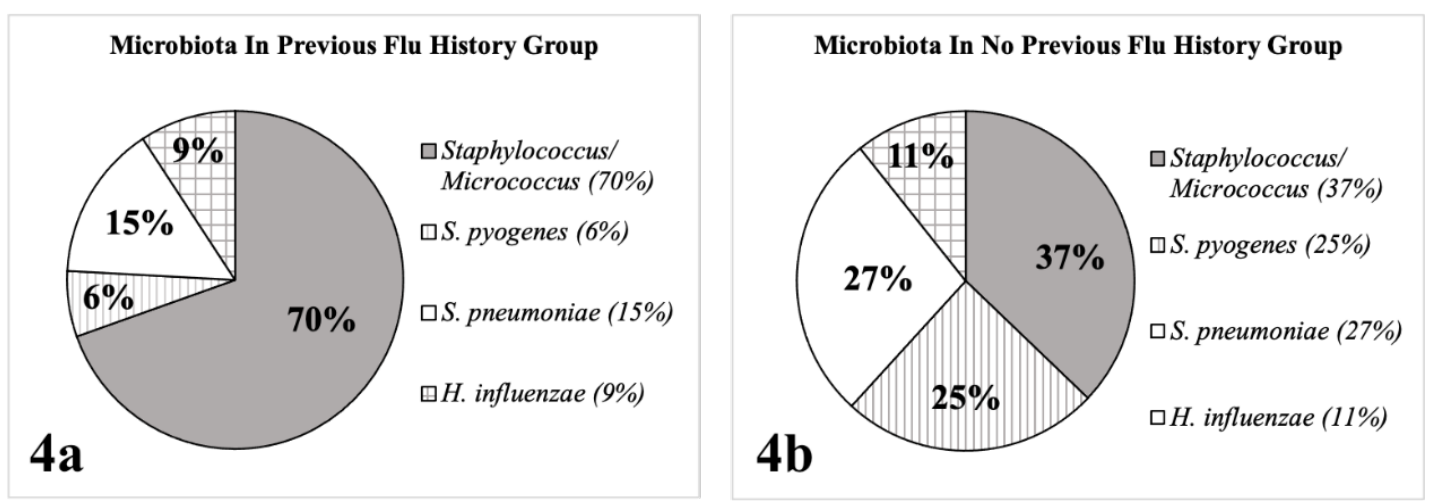

Figure 4. The ratios of the total microbiota, measured as CFU, found in the nasal cavities of those with (a) previous history of influenza and (b) with no previous diagnosis of influenza determined through selective differential agar.

\section{Test to Predict Susceptibility to Influenza Infection: Method 1}

Method 1 analyzed the ratios of Staphylococcus and Micrococcus found in the human nasal cavity. The isolates, based on their bacitracin susceptibility, were categorized as Staphylococcus or Micrococcus for each of the influenza history categories. Once these values were determined and categorized, a Chi-Square Analysis was performed (Table 2). When the frequencies for each genus were compared, individuals without any history of influenza had more Staphylococcus and less Micrococcus than individuals with a previous flu history (Figure 5), supporting the previous assertion in the literature that Staphylococcus may protect against influenza (Chen et al., 2016).

Table 2. Micrococcus and Staphylococcus levels correlate with influenza susceptibility

\begin{tabular}{|c|c|c|}
\hline Bacteria Type & Previous Influenza (n=21) & No Previous Influenza (n=17) \\
\hline Staphylococcus & 51 & 53 \\
\hline Micrococcus & 39 & 14 \\
\hline \multicolumn{2}{r}{ Chi-Square statistic: 8.64} & $\mathrm{p}=0.003$ \\
\hline
\end{tabular}

Table 2. A Chi-Square Analysis was conducted on the values above, resulting in a significant $p$-value $(\mathbf{p}=\mathbf{0 . 0 0 3})$ when comparing number of Staphylococcus and Micrococcus CFU from the previous history of influenza and no previous history of influenza categories, respectively, indicating a correlation of these bacterial types with frequency of influenza illness. There were 90 isolates tested in the previous history of influenza group (out of $>11,000 \mathrm{CFU}$ counted) and 67 isolates tested in the no previous history of influenza group (out of $>1,000 \mathrm{CFU}$ counted). These were plated from a 1:10 dilution, so the original number of bacteria recovered from the swabs is estimated to be 10x higher. Zero CFU grew on negative control plates (not shown), indicating success in following sterile procedure. 

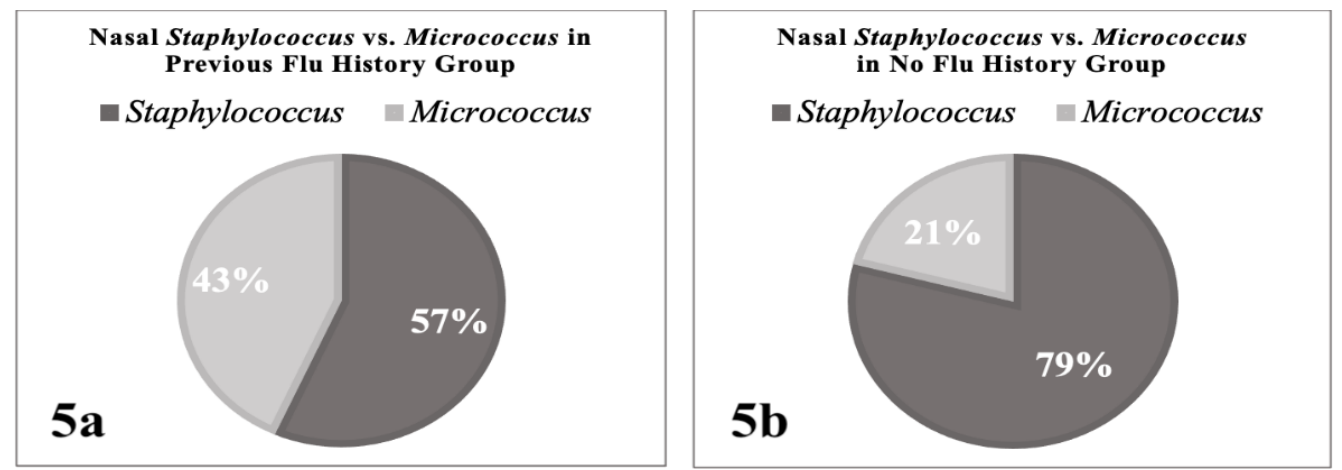

Figure 5. The ratios Micrococcus and Staphylococcus were determined by the disk diffusion test with bacitracin. These ratios were compared for those (a) with previous history of influenza and (b) with no previous diagnosis of influenza.

\section{Test to Predict Susceptibility to Influenza Infection: Method 2}

Method 2 analyzed the ratios of $S$. pyogenes and $S$. pneumoniae found in the human nasal cavity. Based upon the presence or absence of a hemolytic halo, the isolates were determined to be either S. pyogenes or S. pneumoniae for each of the influenza history categories, and a Chi-Square Analysis indicated a significant difference in their numbers between influenza history groups (Table 3). In terms of ratios, there was markedly less S. pyogenes than S. pneumoniae found in individuals with a previous influenza diagnosis, while the ratio was more even in individuals with no previous history of influenza (Figure 6).

Table 3. S. pyogenes and S. pneumoniae levels correlate with influenza susceptibility

\begin{tabular}{|c|c|c|}
\hline Bacteria Type & Previous Influenza $(\mathbf{n = 2 1})$ & No Previous Influenza $(\mathbf{n = 1 7})$ \\
\hline S. pyogenes & 48 & 54 \\
\hline S. pneumoniae & 115 & 60 \\
\hline \multicolumn{2}{|r|}{ Chi-square statistic: 9.26} & $\mathrm{p}=0.002$ \\
\hline
\end{tabular}

Table 3. A Chi-Square Analysis was conducted on the values above, resulting in a significant $\mathbf{p}$-value $(\mathbf{p}=\mathbf{0 . 0 0 2})$ when comparing the average number of $S$. pyogenes and $S$. pneumoniae CFU per participant from the previous influenza and no previous influenza categories, respectively. This indicates that levels of these types of bacteria in a nasal microbiome correlate with frequency of influenza illness. These CFU were plated from a 1:10 dilution, so the original number of bacteria recovered from the nasal swabs is estimated to be 10x higher. Zero CFU grew on negative control plates (not shown), indicating success in following sterile procedure.
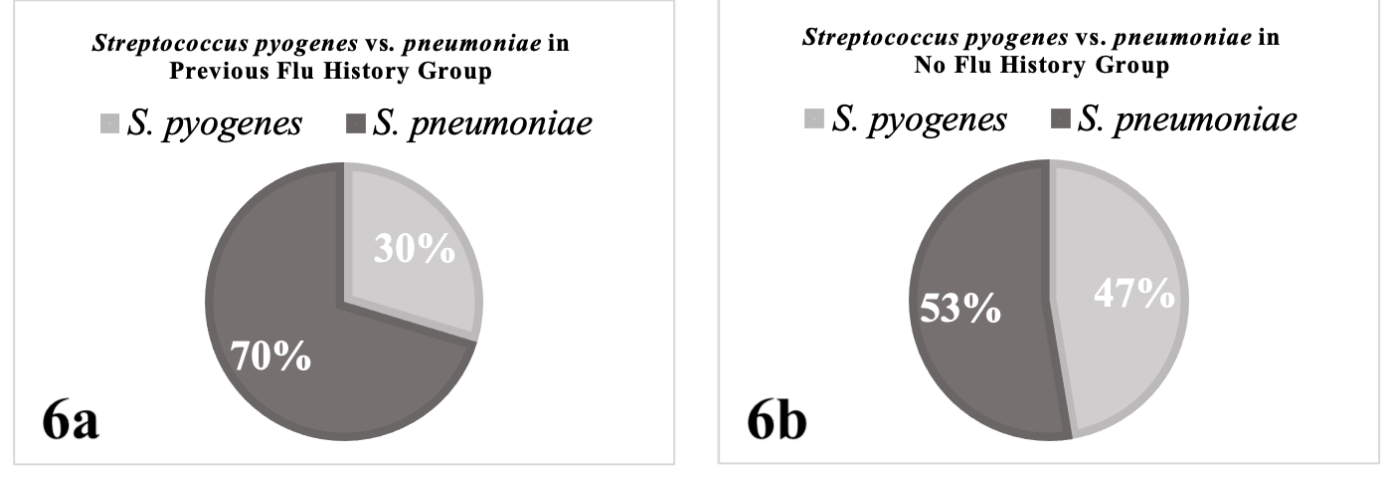
Figure 6. Ratios of S. pyogenes and S. pneumonia were determined by observations of hemolysis on Selective Strep media. These ratios were compared for those (a) with previous diagnosis of influenza and (b) with no previous diagnosis of influenza.

\section{Test to Predict Susceptibility to Influenza Infection: Method 3}

Method 3 analyzed the ratios of Streptococcus spp. and H. influenzae found in the human nasal cavity based on CFU counted on Selective Strep agar and Chocolate agar with bacitracin. A Chi-Square Analysis indicated a significant difference in their numbers between influenza history groups (Table 4). When the frequencies for each type of bacteria were compared, individuals who did not have any history of influenza diagnosis had more Streptococcus spp. and less H. influenzae than individuals with a previous history of influenza diagnosis (Figure 7).

Table 4. Streptococcus and H. influenzae levels correlate with influenza susceptibility

\begin{tabular}{|c|c|c|}
\hline Bacteria Type & Previous Influenza (n=21) & No Previous Influenza $(\mathbf{n = 1 7})$ \\
\hline Streptococcus spp. & 163 & 114 \\
\hline H. influenzae & 71 & 24 \\
\hline \multicolumn{2}{|c|}{ Chi-Square statistic: 7.66} & $\mathrm{p}=0.006$ \\
\hline
\end{tabular}

Table 4. A Chi-Square Analysis was conducted on the values above that yielded a statistically significant $\mathbf{p}$-value (p = 0.006) when comparing number of average CFU per participant from the Selective Strep agar plates (which isolates Streptococcus species) and Chocolate agar plates (which isolates $H$. influenzae) in the previous influenza and no previous influenza categories, respectively. This indicates that levels of these types of bacteria in a nasal microbiome correlate with frequency of influenza illness. These CFU were plated from a 1:10 dilution, so the original number of bacteria recovered from the nasal swabs is estimated to be 10x higher. Zero CFU grew on negative control plates (not shown), indicating success in following sterile procedure.
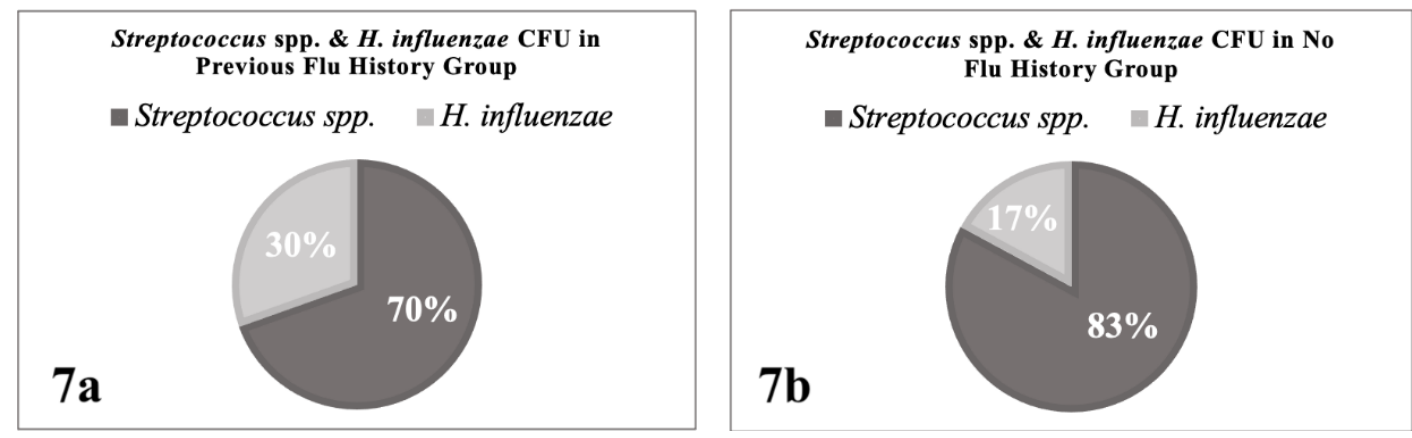

Figure 7. Ratios of Streptococcus spp. and H. influenzae were determined by counting total CFU on the Selective Strep agar and Chocolate agar plates and were compared in groups (a) with previous history of influenza and (b) with no previous diagnosis of influenza.

\section{Comparing Vaccination Participation to History of Influenza Infection}

The survey sent out to participants in this study also inquired about influenza vaccination history as well as previous history of influenza infection. Participants were divided into four groups based on their survey answers: (1) high vaccination and previous influenza, (2) high vaccination and no previous influenza, (3) low vaccination and previous influenza, and (4) low vaccination and no previous influenza. The members of this study were then placed into their respective groups and a Chi-Square Analysis was conducted (Table 5). The p-value for this analysis was not significant $(\mathbf{p}=\mathbf{0 . 4 1 5})$, indicating that students receiving 8 or more flu shots in their lives did not have lower influenza infection rates than those receiving 7 or fewer flu shots. Similar Chi-Square Analyses using different cut-offs for "high 
vaccine" and "low vaccine" (for example, considering "high vaccine" 10+ and "low vaccine" 9 or less; or alternatively, considering any flu vaccination "high vaccine" and limiting "low vaccine" to only those participants who had never been vaccinated) had similar results, all with $\mathrm{p}>0.05$ (analyses not shown).

Table 5. Vaccination status is not predictive of influenza susceptibility

\begin{tabular}{|l|c|c|}
\hline Vaccine History & Previous Influenza $(\mathbf{n = 2 1})$ & No Previous Influenza $(\mathbf{n = 1 7})$ \\
\hline High Vaccine (8+) & 15 & 10 \\
\hline Low Vaccine (0-7) & 6 & 7 \\
\hline \multicolumn{2}{|c|}{ Chi-Square statistic: 0.66} & $\mathrm{p}=0.415$ \\
\hline
\end{tabular}

Table 5. In the study population, rate of influenza vaccination did not correlate with history of influenza contraction according to a Chi-Square Analysis $(\mathbf{p}=\mathbf{0 . 4 1 5})$.

In looking at more differentiated rates of vaccine participation, the number of participants with and without previous influenza history was generally comparable amongst those who had received 0 to 3 flu shots, 4 to 7 flu shots, 8 to 9 flu shots, or 10 or more flu shots in their lifetimes, with perhaps even a higher number of flu shots in those with a previous flu history (Figure 8). When compared to the previous comparisons showing significant differences in nasal microbiome composition between influenza history groups (Tables 1, 2, and 4), this would appear to indicate that one's nasal microbiome may have a greater effect on his or her influenza susceptibility than even vaccination history, at least in this limited study population, an interesting outcome given the wealth of evidence supporting vaccination for influenza control.

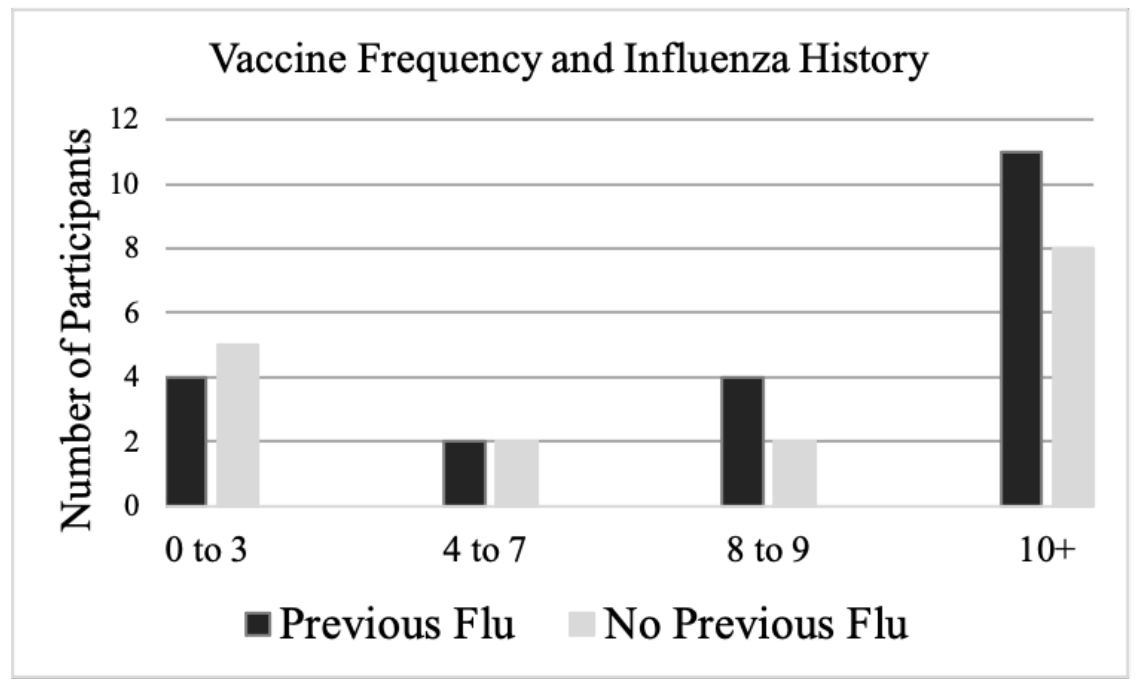

Figure 8. This representation of the data shows further distinction in rates of vaccine participation, with past influenza history similar amongst those who reported 0 to 3,4 to 7,8 to 9 , or $\geq 10$ (10+) influenza vaccinations in their life time.

\section{Discussion and Implications}

The goal of this study was to determine if differences in nasal microbiome exist between individuals with different histories of influenza infection, specifically between those participants who had been diagnosed with influenza by a medical professional at some point in their lives and those who had not. The particular targets of interest among the nasal microbiota, including species of Micrococcus, Staphylococcus, Streptococcus, and Haemophilus, differed in 
their respective numbers and ratios between the two groups of participants $(\mathbf{p}<\mathbf{0 . 0 0 0 0 1})$ (Table 1, Figure 4), with bacteria in the Micrococcus and Staphylococcus category present in lower proportions and the two species of Streptococcus present in higher proportions from nasal swabs of those participants who had never been diagnosed with influenza.

This study developed and assessed three separate methods as potential tools to identify an individual's susceptibility to influenza based on their nasal microbiome composition. Each method involved using basic laboratory skills and supplies, but all are efficient and simple to complete with similar time requirements, except Method 1, which has an added step and an extra incubation period. In Method 1, ratios of Staphylococcus and Micrococcus were compared, in the two influenza history categories, and a significant correlation between these bacterial ratios and a participant's history of influenza infection was found $(\mathbf{p}=\mathbf{0 . 0 0 3})$ (Table 2). Specifically, a higher ratio of Staphylococcus to Micrococcus was found in those without previous influenza history (Figure 5) supporting the hypothesis that stronger colonization of Staphylococcus (at least relative to Micrococcus) may be beneficial in lowering influenza susceptibility, as suggested by others (Chen et al., 2016). Method 1 was a test comprised of multiple plates of one agar type (MSA), bacitracin antibiotic disks, and the supplied nasal swab. These supplies, in addition to basic laboratory supplies like ethanol, gloves, etc., cost \$5.76 per nasal swab. This cost would increase further if more than the 3 to 5 isolates per person used in this study were tested with bacitracin disks.

Method 2 compared the total CFU for two species of Streptococcus, specifically S. pyogenes and S. pneumonia, in both the previous and no previous influenza groups and found that there is a significant difference $(\mathbf{p}=\mathbf{0 . 0 0 2})$ (Table 3). Specifically, S. pneumoniae was observed more than twice as often as $S$. pyogenes in the participants reporting previous influenza diagnoses, while the ratio was more even between the two streptococcal species in those with no previous history of influenza infection (Figure 6), suggesting that an imbalance in these particular species could impact influenza susceptibility. Method 2 involved one Selective Strep agar plate, basic laboratory supplies, and the provided nasal swab. These combined costs come to $\$ 1.89$ per nasal swab. This is the least expensive method, because it uses the least supplies, requiring only one agar plate, one loop, etc.

Method 3 counted total CFU on chocolate agar with bacitracin plates, which select for H. influenzae, and the total CFU, including the combined numbers of Streptococcus pyogenes and Streptococcus pneumoniae, grown on Selective Strep agar. The ratios of these bacterial species were compared in the two different influenza history categories and differed significantly ( $\mathbf{p}=\mathbf{0 . 0 0 6}$ ) (Table 4) with more Streptococcus spp. and less H. influenzae in individuals with no history of influenza diagnosis (Figure 7). This echoed the analysis of the overall nasal microbiome composition where total Streptococcus ratios increased (Figure 4) and total numbers of H. influenzae decreased (Table 1). Method 3 involved one Selective Strep agar plate, one Chocolate agar with bacitracin plate, the nasal swab provided and basic laboratory supplies. This test cost $\$ 4.04$ per nasal swab.

In summary, each of these three methods could be useful in assessing an individual's nasal microbiome and predicting their relative susceptibility to contracting influenza. If one wanted to choose one particular method, Method 2 has the lowest cost and is also the simplest to conduct. However, there is still need for further study before any one of these methods could be pursued for commercialization and widespread use. These methods should be applied to larger sample sizes than the 38 individuals who participated in this study. It should also be determined whether the trends observed in this group apply to other demographics, including those outside of the 15-18 year age range and those with other health conditions, like immunocompromised patients, people with demonstrated allergies to the influenza vaccine, and more. It should also be assessed whether males and females or people from different racial and ethnic backgrounds have different outcomes with these nasal bacteria during an influenza infection. Ideally, a prospective study could be designed that would assess the nasal microbiomes of participants with all three methods described in this study, and then follow them for several years, testing them each year for nasal microbiome composition and for influenza during respiratory illnesses each year, to determine which method most accurately identifies those at greater risk for contracting influenza. Such a study could also assess other species of nasal bacteria through PCR or sequencing methods to determine which species or strains are best associated with low influenza rates. 
Through analysis of participant vaccine participation history (Table 5, Figure 8) and their respective influenza history categories, it appeared that influenza vaccines did not have a significant effect on one's susceptibility to influenza in this study population $(\mathbf{p}=\mathbf{0 . 4 1 5})$. This counterintuitive result may be explained by a number of factors, such as the fact that participants could have contracted influenza only during years they did not get the vaccine, or when they got the vaccine late in the season only after contracting influenza. It is also well known that the strains of influenza included in the vaccine are not always well matched with the strains circulating in a given region and also have narrow scope, with only 2-3 strains included in each year's annual vaccine. It is also possible that a student, after experiencing the undesirable symptoms of influenza one year, may be more likely to get the vaccination in future years. In short, this data should not be taken to mean that influenza vaccination is unimportant. Rather, we hope it will convince the reader that an individual's nasal microbiome could play an even more critical role in defending the body against influenza, particularly in those who don't vaccinate every year.

We also feel it prudent to consider our results as a potential case of "Which came first? The chicken or the egg" - In other words, when assessing influenza rates and nasal microbiome composition, further study is needed to determine the effects these two factors have on each other. Given the published literature suggesting that Staphylococcus may be protective against influenza (Chen et al., 2016), it was interesting to see bacteria in the Micrococcus and Staphylococcus category present in lower proportions in those without a history of influenza diagnosis (Table 1, Figure 4). Further assessment to categorize these bacteria as Micrococcus OR Staphylococcus did indicate that Staphylococcus was more prominent than Micrococcus, despite the overall numbers of these two genera being almost an order of magnitude less in the participant group without previous flu history (Table 1). Overall, our findings suggest that the contribution of Staphylococcus to protection from influenza may be more nuanced than raw numbers and may also require consideration of the overall ratios and balance of different microbiome contributors or of individual Staphylococcus species. In this work, we have mainly considered the effects of certain nasal bacteria in reducing one's susceptibility to influenza. It should also be determined by additional research whether infection with influenza can alter the nasal microbiome composition. For example, perhaps the greater numbers of bacteria in the Staphylococcus and Micrococcus group in participants with a previous influenza diagnosis were higher as a result of that influenza history. In other words, it is conceivable that infection with influenza could have the result of selecting for protective nasal bacteria that increased in number while fighting the influenza virus, and were maintained after the infection was cleared as a protection against future influenza infections. If such alterations in nasal microbiome composition occur with respiratory illness, it would be essential to identify the specific impacts of such infections on various members of the nasal microbiome, as well as whether changes are temporary or permanent in nature, and whether routine vaccination with the live attenuated influenza vaccine available as a nasal spray would have a similar effect on nasal microbiome composition.

Finally, this work suggests that certain species of nasal bacteria may act as a defense against influenza, if they are present in large enough numbers or in proper ratios with other members of the nasal microbiota. This conclusion further suggests that these species, or others identified to be beneficial in this way, could be added to the noses of susceptible individuals to increase the probability that they can offer significant defense to that person. This kind of bioaugmentation procedure in individuals deemed to be at high risk for contracting influenza is not unlike probiotic supplementation or fecal microbiota transfer to enrich for desired gut bacteria species associated with desirable gastrointestinal outcomes. Bioaugmentation of nasal bacteria would entail inoculating the beneficial bacteria into the noses of individuals who show low quantities of these bacteria when tested with the methods described in this paper. This nasal inoculation would encourage the growth of protective bacteria in the nasal microbiome and increase the probability of a shift to the preferred nasal microbiome composition to reduce the likelihood of influenza transmission to these individuals. Such a procedure would likely have a low risk of causing serious complications or side effects because it involves the transfer of or enrichment for commensal bacterium that already regularly inhabit the human nasal surfaces. Strains of desirable species that do not cause disease could also be selected for this reason. Of course, such a procedure would still require clinical trials for safety and efficacy and should not be attempted outside of the 
care of a physician. It should also not be assumed that this would replace the need for routine influenza vaccination, which has a long history of proven success.

\section{Conclusion}

Significant differences in the nasal microbiota were identified in participants with and without previous influenza infection history. Three methods were developed to compare nasal microbiome composition to influenza infection history. Overall, Method 2, comparing two different species of Streptococcus, was the simplest to perform and the most cost effective. Further research should assess whether these findings scale to other populations with more diverse demographics and health histories. In the future, a nasal bioaugmentation therapy could be developed, similar to how certain gastrointestinal conditions are already treated with oral probiotic supplementation, with the goal of increasing the presence of beneficial symbionts in the nasal passages of hosts deemed to have an undesirable nasal microbiome composition prior to such a procedure. In total, this study combined with research already present in the published literature suggests that an individual's nasal microbiome plays an important role in protecting against influenza. Further research to identify the most protective nasal symbionts is needed, along with specifics on the mechanisms through which these bacteria protect their hosts, to support anti-influenza control measures and better protect individuals and global health systems from this burdensome disease.

\section{Limitations}

One limitation was the use of a high school student population with varied self-reported influenza histories, without having information about how recently the last vaccine was received or what type of vaccine was received (such as the live attenuated nasal spray vaccine or the inactivated intramuscular injection vaccine). If this research is expanded, we would ideally have access to a large and diverse sample population with recent and verified influenza infections or verified lack of infection. This study also relied on self-reporting, rather than direct access to medical records, so we cannot be $100 \%$ certain of the accuracy of the participant's memory. It is also possible that infections with influenza that went undiagnosed were not reported in our data set.

Cost was also a factor in certain situations. For example, initial analysis with MSA agar resulted in over 13,000 CFU counted that were either Staphylococcus or Micrococcus. However, due to cost of MSA agar and bacitracin disks, only 157 of these were tested with bacitracin to determine their specific genus, limiting the amount of data available for analysis in Method 1. We also limited our study to 38 participants because of cost limitations in ordering more chocolate agar with bacitracin and selective strep agar plates. Finally, only using bacterial morphology and selective agar types to identify bacteria was a limitation. An alternative would have been isolating multiple bacterial specimens from each sample and sequencing their DNA. Though this method is the most effective at identifying bacteria at the species level, it is also very costly, particularly as this study characterized almost one thousand CFU to genus or species level.

\section{Acknowledgements}

We would like to thank Dr. Patrycja Krakowiak for her wealth of knowledge and willingness to help in aspects of statistical analysis and study design; we could not have done this without her aid and encouragement. Thank you, also, to Mrs. Monica Jaskovic R.N., our school nurse, for the collection of all nasal samples. In addition to these people, we would also like to acknowledge the Capstone program at the Arkansas School for Mathematics, Sciences, and the Arts for the laboratory space, equipment, and resources. 


\section{References}

Agrawal, A., \& Murphy, T. F. (2011). Haemophilus influenzae infections in the H. influenzae type b conjugate vaccine era. Journal of Clinical Microbiology, 49(11), 3728-3732. https://doi.org/10.1128/JCM.05476-11

Baker, J. S., Hackett, M. F., \& Simard, D. J. (1986). Variations in bacitracin susceptibility observed in Staphylococcus and Micrococcus species. Journal of Clinical Microbiology, 23(5), 963-964. https://doi.org/10.1128/JCM.23.5.963-964.1986

Becker, K., Skov, R., \& Von Eiff, C. (2015). Staphylococcus, Micrococcus, and Other Catalase-Positive Cocci. In Manual of Clinical Microbiology (11th ed., pp. 354-382). ASM Press. Chapter doi:10.1128/9781555817381.ch21

Büttner, H., Mack, D., \& Rohde, H. (2015). Structural basis of Staphylococcus epidermidis biofilm formation: mechanisms and molecular interactions. Frontiers in Cellular and Infection Microbiology, 5, 14. https://doi.org/10.3389/fcimb.2015.00014

Chen, H. W., Liu, P. F., Liu, Y. T., Kuo, S., Zhang, X. Q., Schooley, R. T., Rohde, H., Gallo, R. L., \& Huang, C. M. (2016). Nasal commensal Staphylococcus epidermidis counteracts influenza virus. Scientific Reports, 6, 27870. https://doi.org/10.1038/srep27870

Cole, A. M., Dewan, P., \& Ganz, T. (1999). Innate antimicrobial activity of nasal secretions. Infection and Immunity, 67(7), 3267-3275. https://doi.org/10.1128/IAI.67.7.3267-3275.1999

Day, R. L., Harper, A. J., Woods, R. M., Davies, O. G., \& Heaney, L. M. (2019). Probiotics: current landscape and future horizons. Future Science OA, 5(4), FSO391. https://doi.org/10.4155/fsoa-2019-0004

Falk, D., \& Guering, S. J. (1983). Differentiation of Staphylococcus and Micrococcus spp. with the Taxo A bacitracin disk. Journal of Clinical Microbiology, 18(3), 719-721. https://doi.org/10.1128/JCM.18.3.719-721.1983

Haemophilus influenzae. (2018). Retrieved from https://www.cdc.gov/hi-disease/index.html

Johnson, B. A., Anker, H., \& Meleney, F. L. (1945). Bacitracin: A New Antibiotic Produced by a Member of the $B$. subtilis Group. Science (New York, N.Y.), 102(2650), 376-377. https://doi.org/10.1126/science.102.2650.376

Linnes, J. C., Ma, H., \& Bryers, J. D. (2013). Giant extracellular matrix binding protein expression in Staphylococcus epidermidis is regulated by biofilm formation and osmotic pressure. Current Microbiology, 66(6), 627-633. https://doi.org/10.1007/s00284-013-0316-7

Liu, M., Zhao, X., Hua, S., Du, X., Peng, Y., Li, X., Lan, Y., Wang, D., Wu, A., Shu, Y., \& Jiang, T. (2015). Antigenic Patterns and Evolution of the Human Influenza A (H1N1) Virus. Scientific Reports, 5, 14171. https://doi.org/10.1038/srep14171

Marks, L. R., Mashburn-Warren, L., Federle, M. J., \& Hakansson, A. P. (2014). Streptococcus pyogenes biofilm growth in vitro and in vivo and its role in colonization, virulence, and genetic exchange. The Journal of Infectious Diseases, 210(1), 25-34. https://doi.org/10.1093/infdis/jiu058 
Namvar, A. E., Bastarahang, S., Abbasi, N., Ghehi, G. S., Farhadbakhtiarian, S., Arezi, P., Hosseini, M., Baravati, S. Z., Jokar, Z., \& Chermahin, S. G. (2014). Clinical characteristics of Staphylococcus epidermidis: a systematic review. GMS Hygiene and Infection Control, 9(3), Doc23. https://doi.org/10.3205/dgkh000243

Otto M. (2009). Staphylococcus epidermidis--the 'accidental' pathogen. Nature Reviews Microbiology, 7(8), 555567. https://doi.org/10.1038/nrmicro2182

Paharik, A. E., \& Horswill, A. R. (2016). The Staphylococcal Biofilm: Adhesins, Regulation, and Host Response. Microbiology Spectrum, 4(2), 10.1128/microbiolspec.VMBF-0022-2015. https://doi.org/10.1128/microbiolspec.VMBF-0022-2015

Pan, H., Cui, B., Huang, Y. et al. (2016). Nasal carriage of common bacterial pathogens among healthy kindergarten children in Chaoshan region, southern China: a cross-sectional study. BMC Pediatrics 16(161) https://doi.org/10.1186/s12887-016-0703-X

Patterson MJ. (1996) Streptococcus. In: Baron S, editor. Medical Microbiology. 4th edition. Galveston (TX): University of Texas Medical Branch at Galveston; Chapter 13. Available from: https://www.ncbi.nlm.nih.gov/books/NBK7611/

Popova, M., Molimard, P., Courau, S., Crociani, J., Dufour, C., Le Vacon, F., \& Carton, T. (2012). Beneficial effects of probiotics in upper respiratory tract infections and their mechanical actions to antagonize pathogens. Journal of Applied Microbiology, 113(6), 1305-1318. https://doi.org/10.1111/j.1365-2672.2012.05394.x

Rabin, N., Zheng, Y., Opoku-Temeng, C., Du, Y., Bonsu, E., \& Sintim, H. O. (2015). Biofilm formation mechanisms and targets for developing antibiofilm agents. Future Medicinal Chemistry, 7(4), 493-512. https://doi.org/10.4155/fmc.15.6

Reich, N. G., Brooks, L. C., Fox, S. J., Kandula, S., McGowan, C. J., Moore, E., Osthus, D., Ray, E. L., Tushar, A., Yamana, T. K., Biggerstaff, M., Johansson, M. A., Rosenfeld, R., \& Shaman, J. (2019). A collaborative multiyear, multimodel assessment of seasonal influenza forecasting in the United States. Proceedings of the National Academy of Sciences of the United States of America, 116(8), 3146-3154. https://doi.org/10.1073/pnas.1812594116

Rynda-Apple, A., Robinson, K. M., \& Alcorn, J. F. (2015). Influenza and Bacterial Superinfection: Illuminating the Immunologic Mechanisms of Disease. Infection and Immunity, 83(10), 3764-3770.

https://doi.org/10.1128/IAI.00298-15

Warnke, P., Frickmann, H., Ottl, P., \& Podbielski, A. (2014). Nasal screening for MRSA: different swabs--different results!. PloS One, 9(10), e111627. https://doi.org/10.1371/journal.pone.0111627

Wilkins, T., \& Sequoia, J. (2017). Probiotics for Gastrointestinal Conditions: A Summary of the Evidence. American Family Physician, 96(3), 170-178. https://www.aafp.org/afp/2017/0801/p170.html?utm medium $=$ email\&utm source $=$ transaction

Zeng, W., Shen, J., Bo, T., Peng, L., Xu, H., Nasser, M. I., Zhuang, Q., \& Zhao, M. (2019). Cutting Edge: Probiotics and Fecal Microbiota Transplantation in Immunomodulation. Journal of Immunology Research, $2019,1603758$.

https://doi.org/10.1155/2019/1603758 